\title{
Supratentorial Craniotomy Using a Threadwire Saw
}

\section{-Technical Note-}

\author{
Satoru SHIMIZU, Tomoko MiYAZAKI, Sachio SUZUKI, Masaru YAMADA, \\ Satoshi UTSUKI, Hidehiro OKA, and Kiyotaka FUJII \\ Department of Neurosurgery, Kitasato University School of Medicine, \\ Sagamihara, Kanagawa
}

\begin{abstract}
An alternative technique for cutting the bone flap in supratentorial craniotomy uses a threadwire saw (T-saw), originally developed for spinal surgery. After placing a burr hole at each corner of the intended craniotomy, osteotomy is performed between adjacent burr holes using a craniotome, leaving a bony bridge of approximately $1 / 3$ of the length of the osteotomy. The T-saw is introduced between adjacent burr holes through the epidural space and the bridge is cut with reciprocating strokes. The narrow beveled cut reduces the bone gap for fitted bone flap fixation. On closure, the bridge firmly supports the flap and only sutures are needed for fixation. A minimal amount of filler is required to fill the bone gap. Successful bone flap fixation was obtained in more than 100 cases. No technique-related complications such as dural laceration or flap displacement occurred. Osteotomy using a T-saw was somewhat timeconsuming, but cutting efficiency was improved with a Diamond T-saw, featuring a section of cable covered with diamond particles. This method is ideal for bone cuts in cosmetic cranioplasty; is easy and safe to perform, is inexpensive, and avoids the need for flap fixation with metal devices.
\end{abstract}

Key words: craniotomy, cranioplasty, threadwire saw

\section{Introduction}

Craniotomy routinely involves the use of metal fixation systems to secure the bone flaps, for example, screw-fixed titanium plates and bone flap sandwiches fastened between rivet-like titanium clamps. These methods are technically simple, the cosmetic outcomes are satisfactory, and bone flap depression is avoided. ${ }^{4,6,11)}$ However, the design and materials result in high costs, non-uniform surgical techniques, special tools, low flap resistance against compression forces, esthetically objectionable bulges, tenderness of skin over the fixation systems, scalp erosion, screw loosening, migration into the cranium, growth restriction of the skull in children, and artifacts on neuroimaging studies. ${ }^{1,4,6,10,11)}$ Absorbable plates may solve some of these problems, but are expensive and carry the risk of granuloma and sterile abscess formation. ${ }^{2,9)}$ Modified techniques of bone flap fixation without artificial materials, such as bridged craniotomy ${ }^{5}$ and insertion of bone chips as shims, ${ }^{10)}$ provide satisfactory results, but require high levels of technical skill.

We present an alternative simple technique for supratentorial craniotomy using a threadwire saw (T-saw) to cut beveled bone bridges with minimal gaps for fitted bone flap fixation.

\section{Materials and Methods}

We used our new technique in more than 100 adults and children who underwent standard supratentorial craniotomies since April 2006. The craniotomy sites were the forehead and the frontotemporal, temporal, occipital, and high convexity areas. Patients who required emergent removal of the bone flap for decompression were not subjected to this method.

A burr hole is drilled at each corner of the craniotomy and the dura dissected from the inner table. A craniotome is then used for osteotomy between adjacent burr holes. A bony bridge is preserved along approximately $1 / 3$ of the length of

Received July 23, 2007; Accepted December 26, 2007

Author's present address: Satoru Shimizu, M.D., Department of Neurosurgery, Yokohama Stroke and Brain Center, Yokohama, Kanagawa, Japan. 
the osteotomy. Cutting of this bridge with the T-saw is somewhat time-consuming, so the dura was dissected and osteotomy performed at each side just before cutting the bridge to reduce blood loss from the dural surface and the cut edges. The conventional T-saw (Koshiya Medical Instruments, Kanazawa, Ishikawa) used early in this series was later replaced with a Diamond T-saw (MANI, Inc., Utsunomiya, Tochigi). The saw is introduced between the burr holes through the epidural space using a Gigli saw guide (Fig. 1A). If the burr hole is too small to allow
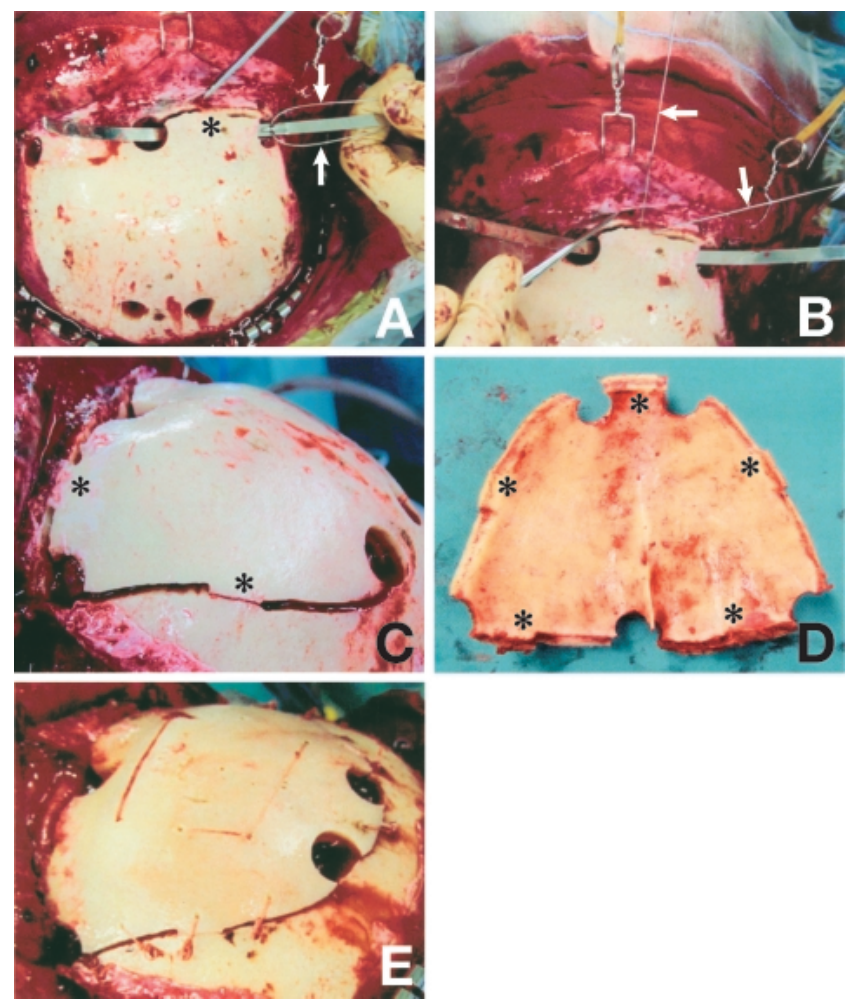

Fig. 1 Photographs of an example of craniotomy using a $\mathrm{T}$-saw in a patient undergoing bifrontal craniotomy. A: A bony bridge (asterisk) is left between two osteotomies performed with a craniotome. The T-saw (arrows) is looped to fit the hook of a Gigli saw guide and passed through the epidural space with the aid of the guide. B: A beveled cut of the bony bridge is made using reciprocating strokes. Arrows indicate the T-saw. C: Completion of the cutting of the bridge. The cut line produced by the T-saw in the bridge (asterisks) is much thinner than that created by the craniotome next to the bridge. D: The removed bone flap shows five bony bridges (asterisks) cut with the T-saw. E: Fitted fixation of the bone flap is obtained with sutures placed at each corner. insertion of the saw guide, a genuine flexible T-saw guide catheter (Koshiya Medical Instruments), a Diamond T-saw guide catheter (MANI, Inc.), or a long flexible needle can be used as the introducer.

The T-saw blade is placed against the edges of the bony bridge, and the ends are held with wire clamps (Koshiya Medical Instruments). Reciprocating strokes, as applied to a Gigli saw, are performed to create a beveled cut (Fig. 1B), which is key for supporting the bone flap on closure. The saw is irrigated with saline solution for cooling during the reciprocating motion. A varying number of bridges are used (Fig. 1C, D); two bridges are adequate, but three or more bridges provide optimum flap stability.

Small drill holes from the surface of the bone to the cut edge at each corner of the craniotomy are used for flap fixation. Non-absorbable 2-0 sutures are passed through adjacent holes in the skull and flap, and tied (Fig. 1E). Tying at the bridge is important to assure flap fixation. Burr hole buttons, methylmethacrylate, and/or bone chips are applied to the burr hole and the bone cut on both sides of the bridge.

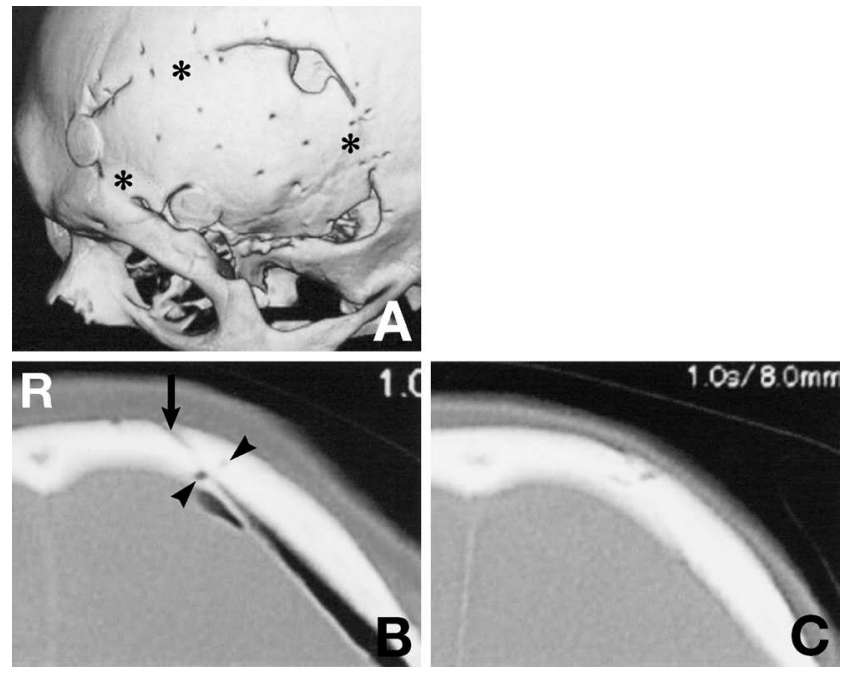

Fig. 2 Postoperative computed tomography scans of a 71-year-old male who underwent left frontotemporal craniotomy using a Diamond T-saw. A: Three-dimensional image 5 months after surgery showing a satisfactory fixation of the bone flap by three wide bony bridges well fused (asterisks). B, C: Axial view of the bony bridge in the forehead just after (B) and 5 months after surgery (C). A thin beveled cut line (arrow) depicted in the former has almost disappeared in the latter after primary bony fusion. Arrowheads show the small drill holes for flap fixation. 


\section{Results}

We could cut bony bridges at any site with either type of T-saw, including the thick anterior skull base and calvarium. The number of bridges was two to six. The procedure was tiring and time-consuming, requiring 1-3 minutes per bridge, using the conventional T-saw without a diamond coating. The introduction of the Diamond T-saw allowed wider bony bridges, ideal for supporting the flap, to be cut easily and rapidly, requiring less than 30 seconds per bridge. No technique-related complications, such as dural laceration, depression, or displacement of the flap, were encountered in the follow-up period of 1-14 months (Fig. 2).

\section{Discussion}

We first applied the present technique to a child with moyamoya disease who underwent a small craniotomy for superficial temporal artery-to-middle cerebral artery bypass. The main purpose was to avoid the use of metal fixation systems that might damage the fragile thin skin after harvesting the donor artery. As we achieved satisfactory results in pediatric patients undergoing bypass surgery, we applied our technique to adults undergoing standard supratentorial craniotomy.

The key to success with our T-saw technique is to cut beveled bony bridges. The thin bone gap associated with cutting of the bridges assured matched bone fixation and primary bone fusion, even at thin corners of the flap, such as those in the temporal region and in children.

The T-saw, developed by Japanese orthopedic surgeons in 1996, ${ }^{7)}$ was introduced for spinal surgery, such as cervical spine laminoplasty to split the spinous process in the midline with minimal bone gaps, and use has continued to increase. ${ }^{3,8)}$ The T-saw consists of a soft, flexible, disposable stainless-steel microcable with $0.36,0.54$, or $0.81 \mathrm{~mm}$ diameter. We used the $0.54-\mathrm{mm}$ saw, which is the most widely used type in spinal surgery. The Diamond T-saw features a $20 \mathrm{~cm}$ section in the mid-portion of the wire that is coated with diamond particles to increase the effectiveness of the blade. The diameter of this portion is $0.64 \mathrm{~mm}$ in medium-sized saws. The wire adjacent to the diamond-coated portion can be used as a conventional T-saw.

Our technique offers several advantages compared to methods using metal flap fixation systems. First, the flap is supported by the bridges, so artificial materials are only required to fill bone gaps, such as burr hole buttons and methylmethacrylate. Another advantage is low cost. A T-saw costs $\$ 100$, a
Diamond T-saw including a guide catheter costs $\$ 158$, and a minimum set of a metal fixation system (2 metal plates and screws for one small craniotomy) costs \$750. Large craniotomies require more metal instruments to sustain the flap. On the other hand, a craniotomy with two to four bridges usually requires only one T-saw or Diamond T-saw by using the full length of the saw gently, i.e. $70 \mathrm{~cm}$ and $80 \mathrm{~cm}$ for the T-saw and Diamond T-saw, respectively.

Our technique is easier than bridged craniotomy using a thin-bladed chisel, ${ }^{5}$ or bone flap fixation using bone chips as shims harvested from the interior edge of the flap. ${ }^{10)}$ The tip of the chisel may be difficult to see at the dural surface, especially if the skull is thick. The direction of the bone cut with a T-saw, from the dural surface to the skull, is safer than use of a chisel. In addition, the wider bony bridges created by the T-saw are ideal for supporting the flap. Secure fixation of flaps with shims also requires high skill and expertise.

The disadvantages of the T-saw without diamond coating are possible fatigue of the operator's arm and lower cutting speed than the Gigli saw due to the smooth surface of the threadwire. The introduction of the Diamond T-saw solved these problems. Difficulties related to creating a curved cut line with the T-saw are solved by the adjacent osteotomies, in which the craniotome can be used to create the desired cut line(s).

The beveled bone flaps formed by the T-saw, especially the Diamond T-saw, are very useful in supratentorial craniotomy, by facilitating the safe, secure fitting of the bone flap. The method is technically easy, safe, and involves minimal additional costs.

\section{References}

1) Duke BJ, Mouchantat RA, Ketch LL, Winston KR: Transcranial migration of microfixation plates and screws. Case report. Pediatr Neurosurg 25: 31-35, 1996

2) Eppley BL, Sadove AM, Havlik RJ: Resorbable plate fixation in pediatric craniofacial surgery. Plast Reconstr Surg 100: 1-13, 1997

3) Kawahara N, Tomita K, Shinya Y, Matsumoto T, Baba H, Fujita T, Murakami H, Kobayashi T: Recapping T-saw laminoplasty for spinal cord tumors. Spine 24: 1363-1370, 1999

4) Lerch KD: Reliability of cranial flap fixation techniques: Comparative experimental evaluation of suturing, titanium miniplates, and a new rivet-like titanium clamp (CranioFix): Technical note. Neurosurgery 44: 902-905, 1999

5) Ochiai C, Okuhata S, Yoshimoto Y, Nagai M: Bridged craniotomy for stable fixation of a bone flap. J Neu- 
rosurg 85: 518-519, 1996

6) Persing JA, Posnick J, Magge S, Spinelli HM, Wolfe SA, Munro I, Mulliken JB: Cranial plate and screw fixation in infancy: An assessment of risk. J Craniofac Surg 7: 267-270, 1996

7) Tomita K, Kawahara N: The threadwire saw: A new device for cutting bone. J Bone Joint Surg Am 78: 1915-1917, 1996

8) Tomita K, Kawahara N, Toribatake Y, Heller JG: Expansive midline T-saw laminoplasty (modified spinous process-splitting) for the management of cervical myelopathy. Spine 23: 32-37, 1998

9) Weisberger EC, Eppley BL: Resorbable fixation plates in head and neck surgery. Laryngoscope 107: 716-719, 1997

10) Winston KR, Wang MC: Cranial bone fixation: Review of the literature and description of a new procedure. J Neurosurg 99: 484-488, 2003

11) Yaremchuk MJ, Posnick JC: Resolving controversies related to plate and screw fixation in the growing craniofacial skeleton. J Craniofac Surg 6: 525-538, 1995

Address reprint requests to: Satoru Shimizu, M.D., Department of Neurosurgery, Yokohama Stroke and Brain Center, 1-2-1 Takigashira, Isogo-ku, Yokohama, Kanagawa 235-0012, Japan.

e-mail: Satoru4756@aol.com

\section{Commentary}

The authors reported a technical method of supratentorial craniotomy using a diamond thread wire saw
(T-saw) originally developed for spinal surgery by Tomita and Kawahara in 1996. They performed successful craniotomies and bone flap fixations without technique-related complications in more than 100 cases with this technique.

Back in 1894, Gigli first described a simple wire-like device for cutting bone. The Gigli saw was essentially a twisted stainless steel cable with interposed notches, which provides a very effective cutting surface in a reciprocating motion. Thereafter, the Gigli saw and its derivatives have been widely used for various surgical procedures. However, the diameter of Gigli saw is relatively thick and may make a depressed area on the scalp along the craniotomy line caused by the bony defect between the bone flap and the skull edge. Even with craniotomy using electrical or pneumatic high speed drill saws, many patients might have suffered from cosmetic problems after surgery due to the large bony defect along the craniotomy line especially in patients who underwent frontal craniotomies.

To overcome the possible fatigue of the operators and lower cutting speed, the authors coated the saw with diamond particles. The final diameter of diamond thread wires coated on the medium-sized (0.54-mm) T-saws was $0.64 \mathrm{~mm}$. I believe craniotomy using diamond T-saws is an excellent alternative technique for cutting the bone flap in the skull in terms of safety and cosmetic issues.

Hee-Won Jung, M.D. Department of Neurosurgery Seoul National University Hospital Seoul, R.O.K. 